\title{
Semiautomated Clone Verification by Real-Time PCR Using Molecular Beacons
}

BioTechniques 29:1296-1308 (December 2000)

\author{
R.C.A.A. van Schie, S.A.E. \\ Marras $^{1}$, J.M. Conroy, N.J. \\ Nowak, J.J. Catanese, and \\ P.J. de Jong \\ Roswell Park Cancer Institute, \\ Buffalo and 'Public Health \\ Research Institute, New York, \\ NY, USA
}

\begin{abstract}
Conventional, high-throughput PCR analysis of common elements utilizing numerous primer sets and template DNA requires multiple rounds of PCR to ensure optimal conditions. Laborious gel electrophoresis and staining is then necessary to visualize am plification products. We propose novel multicolor molecular beacons, to establish a highthroughput, PCR-based sequence tagged site (STS) detection system that swiftly and accurately confirms marker content in template containing common repeat elements. A sim ple, one-tube, real-time PCR assay system was developed to specifically detect regions containing CA and GATA repeats. Ninetysix samples can be confirmed for marker content in a closed-tube format in $3 \mathrm{~h}$, elim inating product confirmation on agarose gels and avoiding crossover contamination. Multiple STSs can be detected simultaneously in the same reaction tube by utilizing molecular beacons labeled with multicolor fluorophores. Template DNA from 260 RPCI-11 bacterial artificial chromosome (BAC) clones was examined for the presence of CA and/or GATA repeats using molecular beacon PCR and compared with conventional PCR results of the same clones. Of the 205 clones containing $C A$ and GATA repeats, we were able to identify 129 clones $(C A, \mathrm{n}=99 ;$ GATA, $\mathrm{n}=30$ ) by using molecular beacons and only 121
\end{abstract}

clones $(C A, \mathrm{n}=92 ;$ GATA, $\mathrm{n}=29)$ by conventional PCR amplification. As anticipated, 55 clones that contained sequences other than CA or GATA failed molecular beacon detection. Molecular beacon PCR, employing beacons specific for tandem repeat elements, provides a fast, accurate, and sensitive multiplex detection assay that will expedite verification of marker content in a multitude of template containing these repeats.

\section{INTRODUCTION}

Expeditious technological progression in molecular genetics, primarily driven by the Mouse and Human Genome Project, has resulted in a multitude of high-throughput efforts to construct chromosome-specific maps. These maps are based on sequencetagged sites (STSs) (6) and gene-based sequence tagged site markers (ESTs) (5). To date, a physical map consists of a series of well-spaced STSs and covers almost the entire human genome. By positioning ESTs relative to microsatellite markers, a human transcript map is being generated across the genome by the Radiation Hybrid Mapping Consortium (15). The bacterial artificial chromosome (BAC) has proved to be a good cloning vehicle to generate relatively stable libraries (16). Recently, by improving the construction of BAC libraries, Osoegawa et al. (13) were able to create a highly recombinant, 25.3fold redundant human male BAC library (RPCI-11).

Currently, our laboratory is in the process of assembling a genome-wide arrayed BAC clone resource for fluorescence in situ sequence hybridization (FISH) analysis of chromosomes (12).
Individual positive BAC clones are identified through hybridization-based screening of high-density filter sets. At the present time, fluorescent fingerprint analysis (4) and PCR analysis (11) are being used as the standard methodologies to confirm positively selected BAC clones. However, an initiative like this requires technologies that simultaneously advance the output and reduce costs. Above all, the conventional agarose gel electrophoresis technique used to visualize individual PCR products leaves room for improvement because this procedure is very time consuming and often ambiguous. PCR products screened on agarose gels can be vague, and the use of different primer pairs under similar PCR conditions can easily result in the formation of nonspecific PCR products. Furthermore, an undesirable consequence of opening test tubes is the possible PCR product contamination of untested samples (8).

In this paper, we report the development of a semiautomated assay to achieve high-throughput screening. This extremely sensitive multiplex assay eliminates the labor-intensive and time-consuming product confirmation of gel electrophoresis. In a real-time PCR, STS/EST regions are amplified in selected positive BAC clones in sealed reaction tubes in the presence of fluorescent reporter probes. These fluorescent reporter probes, or molecular beacons, can identify specific STS regions. Additionally, an extra level of specificity is being introduced with these molecular beacons because the generation of a fluorescent signal is exclusively due to the detection of the intended PCR product.

Molecular beacons are single- 
stranded hybridization probes that form a stem-and-loop structure and possess an internally quenched fluorophore (19). A nonfluorescent quencher is covalently linked to the $3^{\prime}$ end, and a fluorophore is covalently attached to the $5^{\prime}$ end. In the absence of target, the hairpin stem keeps the fluorophore in close proximity to the quencher, causing fluorescence to be quenched. However, when the probe sequence in the loop anneals to its target, the rigidity of the probe-target helix forces the hairpin stem to unwind, separating the fluorophore from the quencher and restoring fluorescence (Figure 1). Interestingly, structured DNA probes such as molecular beacons show an enhanced specificity in probe-target duplex for- mation (1). In addition, molecular beacons can possess multicolor fluorophores (18), as a result of which different targets can be simultaneously detected in the same solution.

In this report, we describe an innovative test in which multiple short tandem repeats (STRs) are detected in an extremely sensitive high-throughput multiplex detection assay using multicolor molecular beacons.

\section{MATERIALS AND METHODS}

\section{Template DNA}

Template for molecular beacon PCR analysis was BAC clone DNA pre- viously assembled for analysis by conventional PCR. These clones were derived from our RPCI-11 human male BAC library (see http://www.chori.org/ bacpac/11framehmale.htm) as part of an ongoing high-throughput screening project to obtain an ordered set of reagents for FISH. Hybridization positive clones were cultured and prepped for PCR verification of marker content (unpublished data). Whole genomic control DNA was isolated from transformed lymphoblasts obtained from healthy volunteers at the Roswell Park Cancer Institute.

\section{Library Screening Strategy}

For the library screening, we fol-

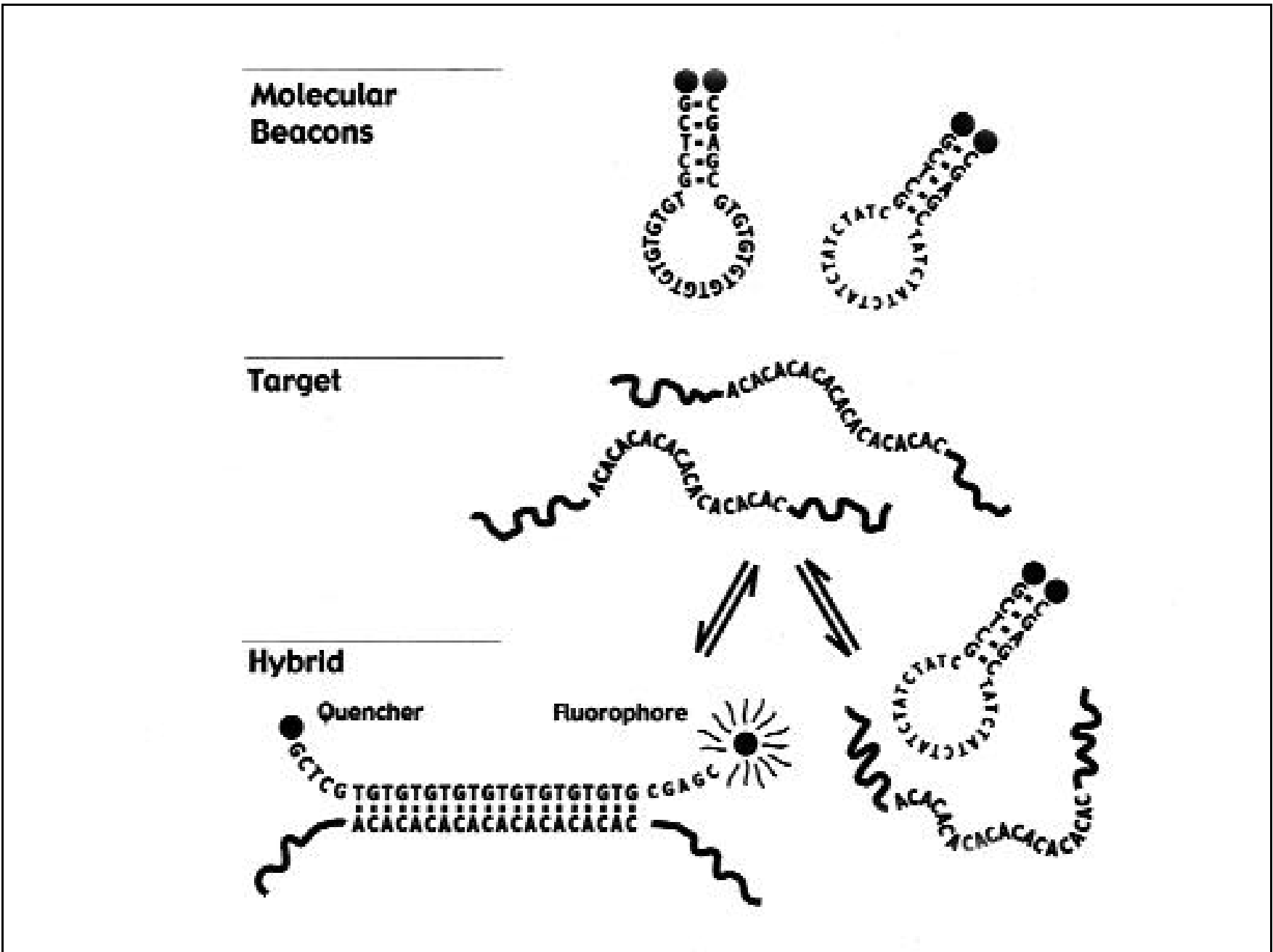

Figure 1. Molecular beacon target-dependent fluorescence. Molecular beacons do not fluoresce in the absence of target because the fluorophore and nonfluorescent quencher are in very close proximity. Annealing of the specific probe sequence (GT repeat) in the loop of a molecular beacon to a complementary target (CA repeat) leads to a conformational reorganization that switches on the fluorescence from the dye present at the $5^{\prime}$ end of the oligonucleotide probe. On the other hand, this specific target-dependent fluorescence will fail to occur with a TATC repeat molecular beacon. 


\section{Research Report}

lowed a previously described strategy (12). To improve the success rate of the marker to BAC correlation, we are em ploying overlapping oligonucleotide probes ("overgo") based on the EST and STS sequences for the markers (10). The overgos are designed with the same average melting characteristics and are labeled by replicating the $5^{\prime}$ overhangs using ${ }^{32} \mathrm{P}$ nucleotide triphosphates. To increase the throughput of the screening process to high-density BAC colony membranes, the probes are pooled in mixtures of 36 probes each. Informative probe mixtures are prepared through the use of a $3-D(6 \times 6 \times$ $6)$ probe pooling strategy consisting of 216 distinct probes.

\section{Selection of PCR Primer Sets}

A Basic Local Alignment Search Tool (BLAST) search was conducted using a UNIX ${ }^{\circledR}$-based script, which automates the sequence analysis to mask repetitive elements (Repeat Masker). PCR of STS/EST marker sequences present in BAC clone DNA was performed using established primer pairs (MapPairs ${ }^{\circledR} ; \quad$ Research Genetics, Huntsville, AL, USA) flanking these markers and generating products ranging from 127 to $299 \mathrm{bp}$.

\section{Synthesis of Molecular Beacons}

To confirm predicted probe-BAC

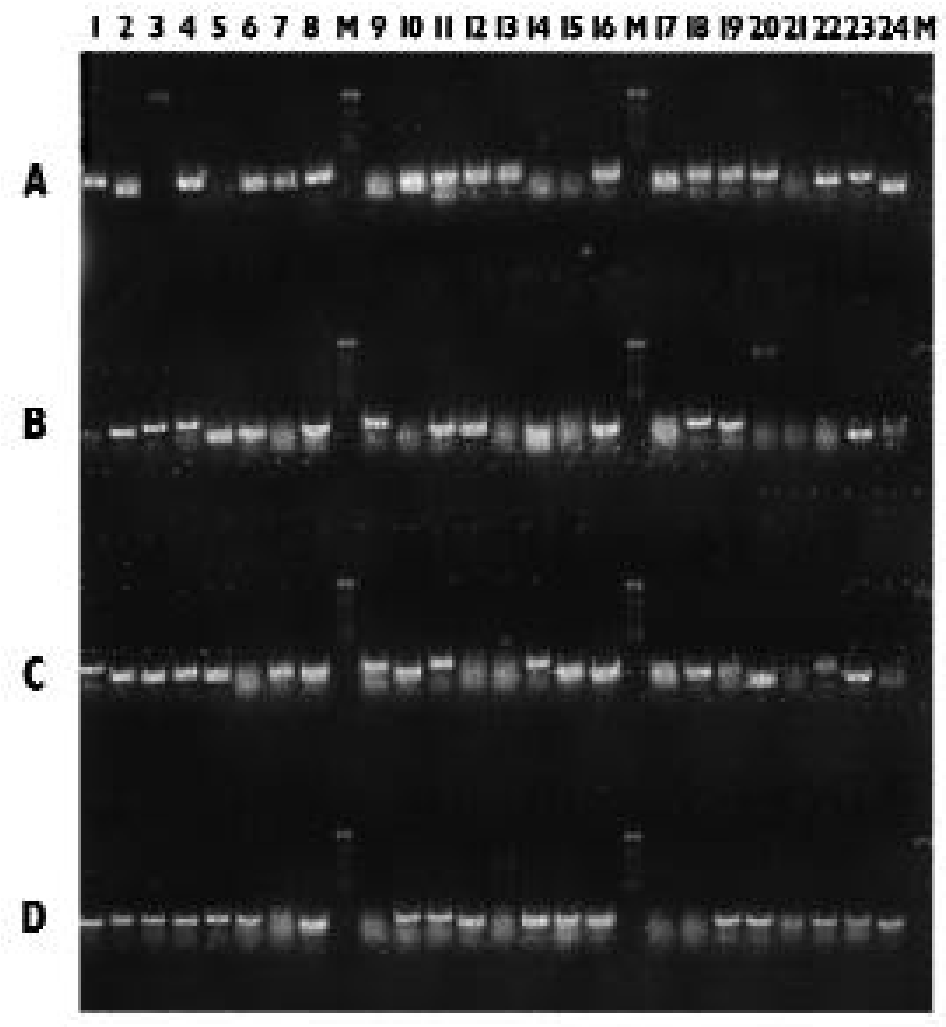

Figure 2. BAC clone confirmation through size assessment of PCR products by agarose gel electrophoresis. This figure shows conventional PCR products of 96 randomly selected samples on a $1.5 \%$ agarose gel, stained with ethidium bromide and visualized under UV illumination. It illustrates that when clones are screened on agarose gels, bands can be rather vague (e.g., row A, lanes 15 and 21), or more than one product has been formed (e.g., row $\mathrm{C}$, lane 9; row D, lane 15), which makes interpretation of the results difficult. Lanes M, 100-bp ladder (Life Technologies, Rockville, MD, USA).

pairs, molecular beacons were constructed capable of recognizing CA or GATA STRs. Synthesized molecular beacons contain two complementary 5nucleotide-long arms and a 20-nucleotide-long probe sequence. For the single-beacon assays (optimalization experiments), fluorescein (FAM) was selected as the fluorophore. To distinguish the fluorescence of one molecular beacon from another in a duplex assay, tetrachloro-6-carboxyfluorescein (TET) and carboxytetramethylrhodamine (TMR) were selected on the basis that their emission maxima are well spaced from each other across the visible spectrum. The fluorophores FAM and TMR (Molecular Probes, Eugene, OR, USA) are joined to a sulphydryl group at their $5^{\prime}$ terminal by removing the protective trityl moiety. The quencher $4-\left(4^{\prime} \mathrm{di}-\right.$ methylaminophenylazo)benzoic acid (DABCYL) (Molecular Probes) is coupled to a primary amino group at its $3^{\prime}$ end utilizing an amine-reactive derivative of DABCYL. The TET molecular beacon was synthesized completely on a DNA synthesizer (Applied Biosystems, Foster City, CA, USA) utilizing a controlled-pore glass column (Glen Research, Sterling, VA, USA) to introduce a 4-dimethylaminoazobenzene-4'-sulfonyl group (DABSYL) at the $3^{\prime}$ end of the oligodeoxyribonucleotide and a tetrachloro-6-carboxyfluorescein phosphoramidite (Glen Research) to introduce the fluorophore at the $5^{\prime}$ end of the molecule. Each molecular beacon was purified by HPLC. A previously described, detailed protocol for synthesizing molecular beacons is available at http://www.molecular-beacons.org.

\section{Nucleotide Sequence of Molecular Beacons}

CA repeat-specific molecular beacons: fluorescein-5' ${ }^{\prime}$ CGAGCGTGTGTGTGTGTGTGTGTGTGCTCG 3'-DABCYL (CA-FAM), carboxytetramethylrhodamine-5' -CGAGCGTGTGTGTGTGTGTGGTGT GCTCG-3' DABCYL (CA-TMR); GATA repeatspecific molecular beacons: fluorescein-5' ${ }^{\prime}$ - CGAGCTATCTATCTATCTAT CTATCGCTCG 3'-DABCYL (GATAFAM), tetrachloro-6-carboxyfluorescein$5^{\prime}$ - CGAGCTATCTATCTATCTATTATCECTCG-3'-DABSYL(GATA-TET), 


\section{Research Report}

where underlines identify the complementary arm sequences of the stem.

\section{Conventional PCRs}

For the standard PCR analysis a PTC-200 ${ }^{\mathrm{TM}}$ thermal cycler (MJ Research, Watertown, MA, USA) was used. PCR mixtures prepared for the conventional amplification reactions did not include molecular beacons. Each 50$\mu \mathrm{L}$ reaction contained 50 ng DNA, 0.20 $\mu \mathrm{M}$ of a specific MapPairs primer set, $250 \mu \mathrm{M}$ deoxy-adenosine triphosphate (dATP), $250 \mu \mathrm{M}$ dCTP, $250 \mu \mathrm{M}$ dGTP, $250 \mu \mathrm{M}$ dTTP (Roche Molecular Biochemicals, Indianapolis, IN, USA), 0.3 U Taq DNA polymerase (Fisher Scientific, Pittsburgh, PA, USA), $1.75 \mathrm{mM}$ $\mathrm{MgCl}_{2}, 50 \mathrm{mM} \mathrm{KCl}$, and $10 \mathrm{mM}$ Tris$\mathrm{HCl}(\mathrm{pH} 8.0)$. The thermal cycling program started with a 4-min denaturation at $95^{\circ} \mathrm{C}$, followed by 30 cycles of amplification (denaturation at $95^{\circ} \mathrm{C}$ for $15 \mathrm{~s}$, annealing at $55^{\circ} \mathrm{C}$ for $30 \mathrm{~s}$, and polymerization at $72^{\circ} \mathrm{C}$ for $45 \mathrm{~s}$ ) and 1 cycle at $72^{\circ} \mathrm{C}$ for $10 \mathrm{~min}$. PCR products were analyzed by agarose gel electrophoresis.

\section{Molecular Beacon PCRs}

Amplification reactions employing molecular beacons were performed in an ABI PRIS $M^{\circledR} 7700$ spectrofluorometric thermal cycler using sealed optical tubes in a 96-well microplate (both from Applied Biosystems). Preceding the PCRs with molecular beacons in duplex, reference emission spectra of CA-TET and GATA-TMR were stored in the spectrofluorometric thermal cycler by hybridizing each molecular beacon to an excess of perfectly complementary oligonucleotides at $55^{\circ} \mathrm{C}$. This enables the generated fluorescent emission spectra to be separated into the contributions from each of the two differently colored molecular beacons present in the same reaction tube.

Each $50-\mu \mathrm{L}$ reaction contained 0.20 $\mu \mathrm{M}$ of a specific MapPairs primer set, $250 \mu \mathrm{M}$ dATP, $250 \mu \mathrm{M}$ dCTP, $250 \mu \mathrm{M}$ dGTP, $250 \mu \mathrm{M}$ dTTP, 2 U AmpliTaq Gold $^{\circledR}$ DNA polymerase (Applied Biosystems), $4 \mathrm{mM} \mathrm{MgCl} 2,50 \mathrm{mM}$ $\mathrm{KCl}, 10 \mathrm{mM}$ Tris-HCl (pH 8.0), $50 \mathrm{ng}$ target DNA, $0.2 \mu \mathrm{M}$ CA-FAM, or 0.2 $\mu \mathrm{M}$ GATA-FAM. When assays were performed in duplex, both beacons were added to the same reaction $(0.2 \mu \mathrm{M}$ CATET and $0.4 \mu \mathrm{M}$ GATA-TMR). The thermal cycling program started with 10 min at $95^{\circ} \mathrm{C}$, by which the AmpliTaq Gold DNA polymerase is activated, followed by 35 cycles of $30 \mathrm{~s}$ at $95^{\circ} \mathrm{C}, 60 \mathrm{~s}$ at $55^{\circ} \mathrm{C}$, and $30 \mathrm{~s}$ at $72^{\circ} \mathrm{C}$. Fluorescence was monitored with a $100-\mathrm{ms}$ exposure time during the annealing steps.

\section{RESULTS}

\section{Clone Confirmation through Conventional PCR}

Positive clones from segment 1 of the RPCI-11 human male BAC Library that had been identified on high-density filter sets were subsequently individually confirmed by PCR analysis. PCRs were performed using unique established primer pairs flanking each of the positive screened STS/EST markers. Clones that contained an EST, a CA, GATA, or GGAA repeat were verified. A total of
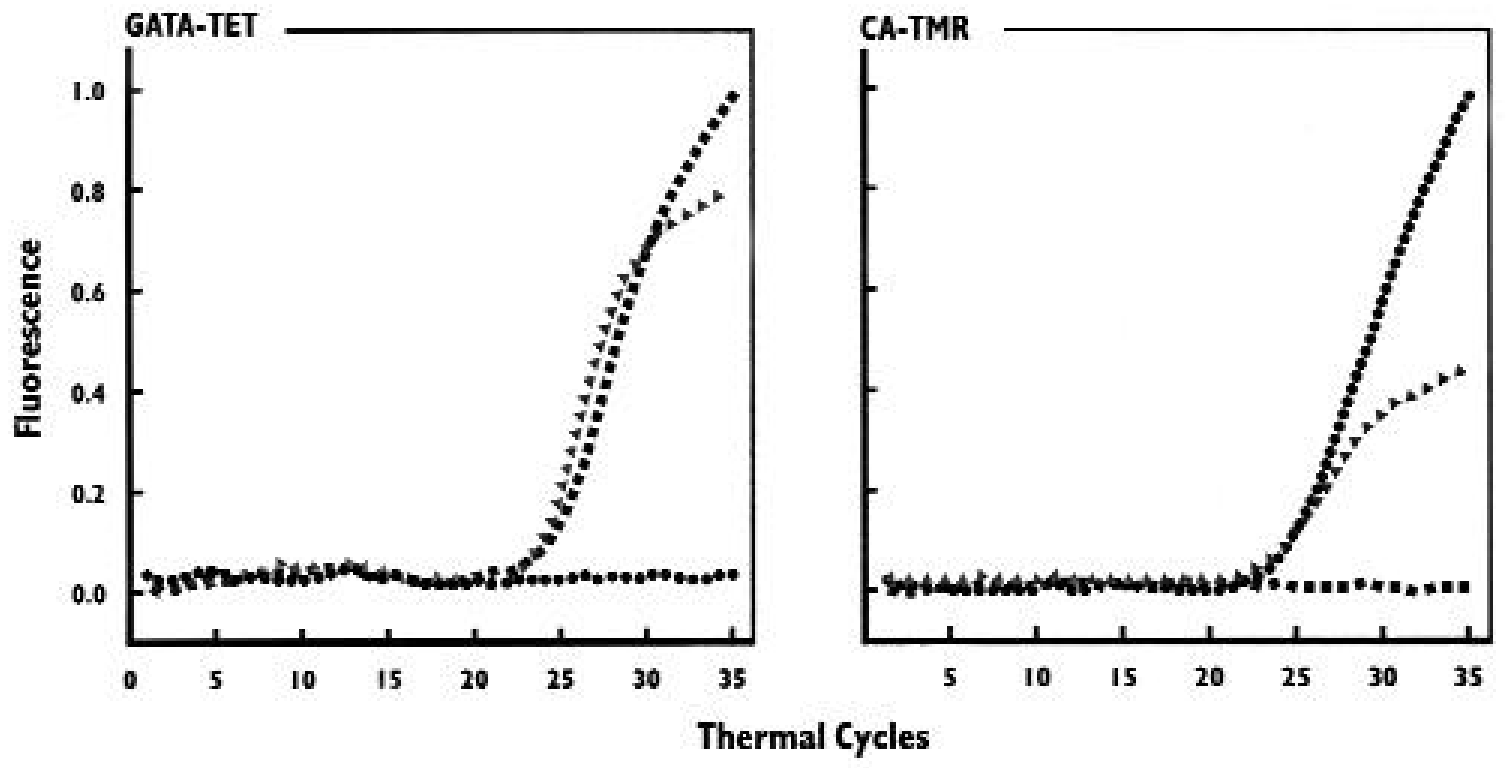

Figure 3. Specificity of real-time multiplex detection of CA and GATA repeat products. Two sets of five assays were carried out in parallel, each initiated with BAC clone DNA containing CA repeats $(\bullet)$, GATA repeats $(\bullet)$, CA/GATA repeats $(\boldsymbol{\bullet})$, BAC clone DNA without any CA/GATA repeats (not shown), and a nontemplate control (not shown). Every tube contained a set-specific established primer pair to amplify either GATA or CA repeats, respectively. All tests were carried out in the presence of two differently colored, STS-specific molecular beacons [GATA-TET, left-hand panel (reports emission at $535 \mathrm{~nm}$ ); CATMR, right-hand panel (reports emission at $575 \mathrm{~nm}$ )]. Only the molecular beacon whose probe sequence was complementary to a product formed a hybrid and emitted a fluorescent signal corresponding to either a CA or GATA repeat. Fluorescence was measured every cycle and plotted versus the cycle number. Fluorescent signals were absent in the template with no repeats and nontemplate control assays (not shown). 


\section{Research Report}

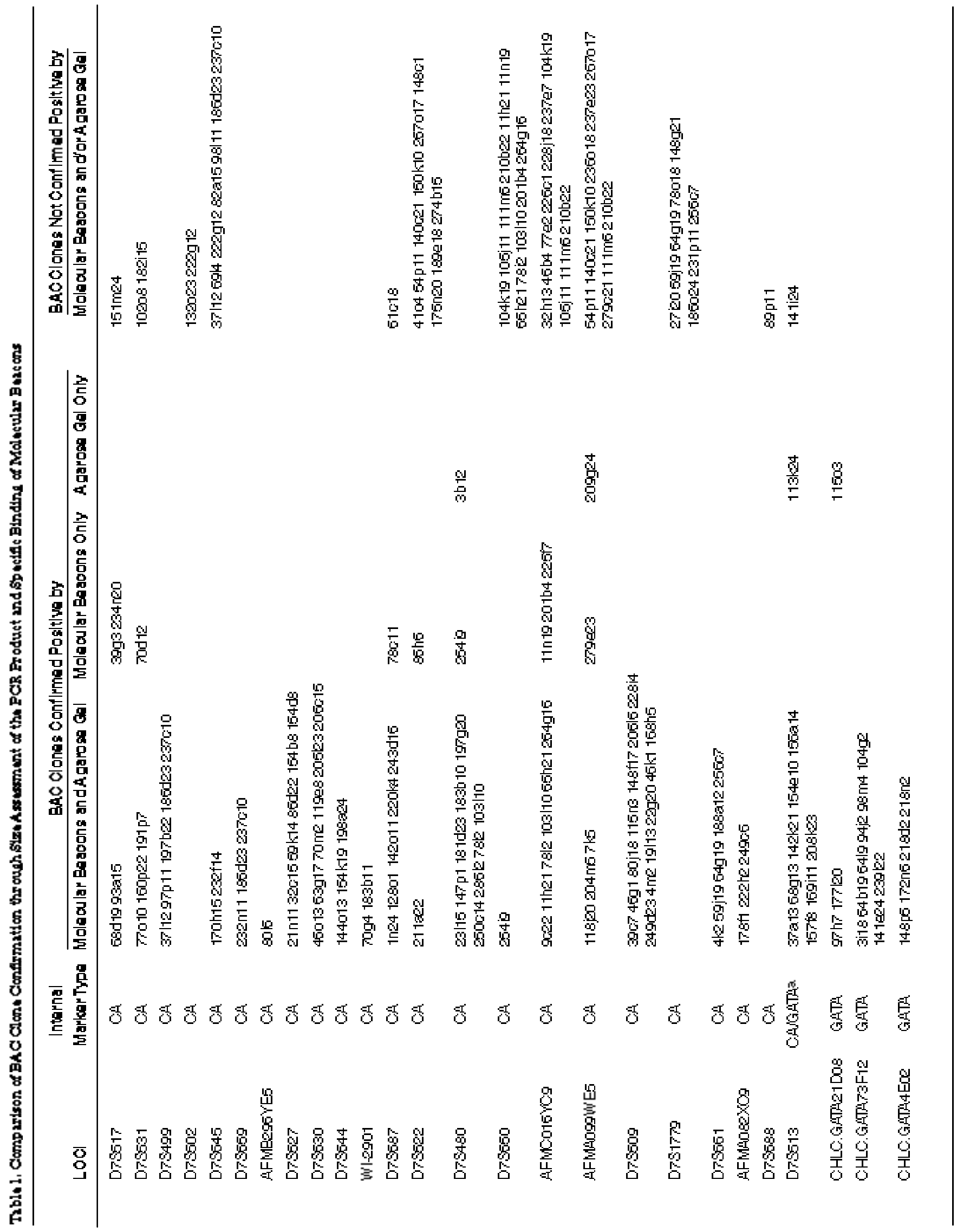




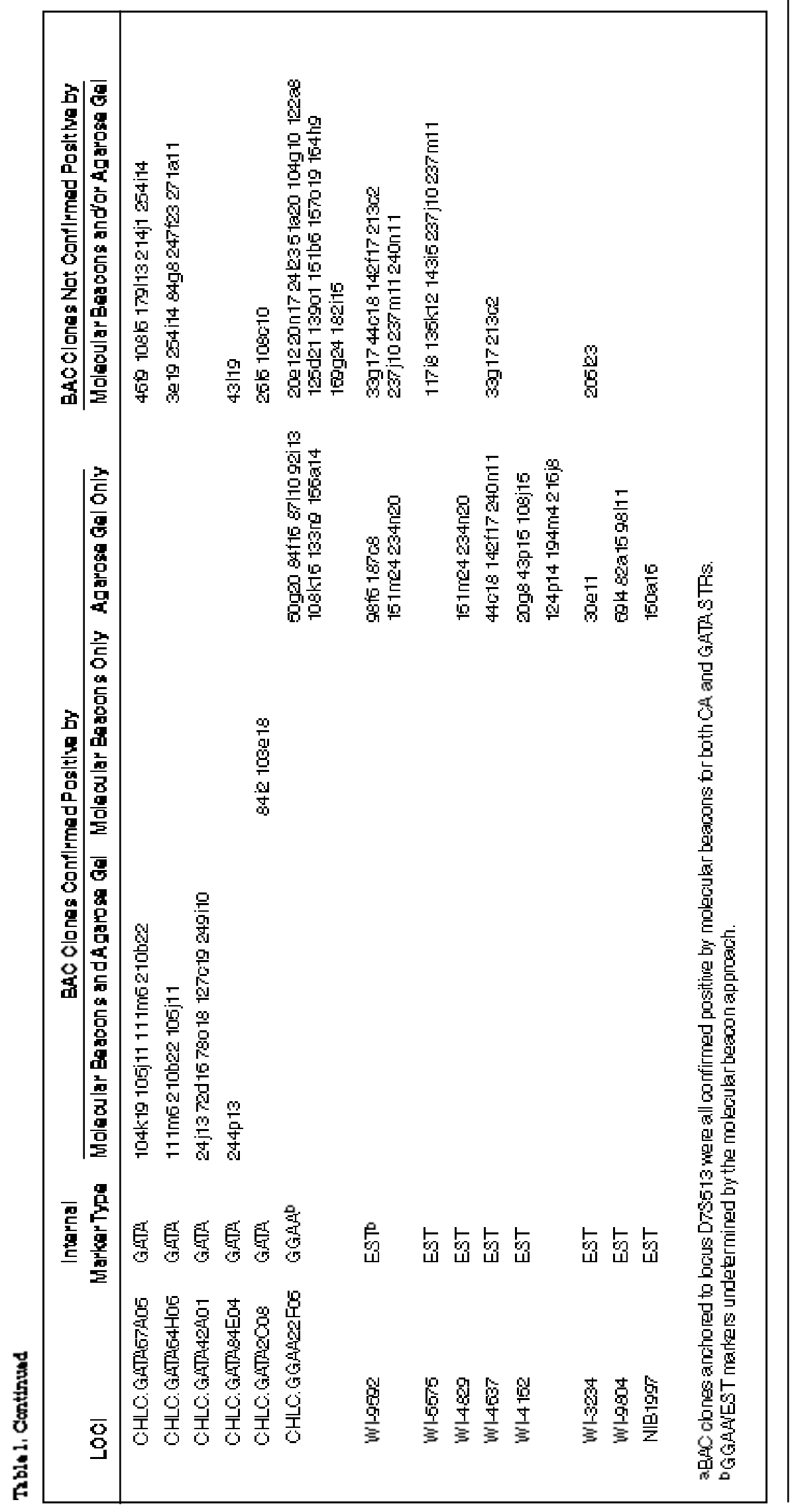


260 BAC clones were tested utilizing 40 different specific oligonucleotide sets derived from as many STS/EST markers available through the Whitehead Center for Genome Research (Cambridge, MA, USA). Out of the 260 hybridization positive clones, $79.2 \%(n=206)$ were unique clones from which 145 clones were verified by conventional PCR and agarose gel electrophoresis (i.e., 70.4\% of the unique clones) (Table 1). Figure 2 shows products of the final confirmation achieved through size assessment of PCR products by agarose gel electrophoresis. As a consequence of the high-throughput screening, the PCR analyses for all STSs were carried out under identical conditions, resulting sometimes in rather vague bands and/or multiple products. This makes interpretation of the results often difficult, and repetitive screening of these clones is therefore inevitable.

\section{Real-Time Detection of PCR Products}

To overcome the imperfections described above of the BAC clone verification through size assessment of PCR products by agarose gel electrophoresis, a semiautomated assay was developed with the ability to identify specific STRs. In a real-time PCR, we detected these STS/EST regions utilizing molecular beacons. First, we had to determine that molecular beacons could easily discriminate between the different STRs. Therefore, two fluorescein-labeled molecular beacons were synthesized, each with internal loop sequences of $20 \mathrm{bp}$ long, complementary to either $10 \mathrm{CA}$ repeats or 5 GATA repeats. The arm sequences of the beacons were designed to form a hybrid at the PCR annealing temperature. To confirm the hairpin structure, a computer program (22) further analyzed the complete sequence of each molecular beacon. For this occasion, established primer pairs for each of the two STRs were selected on the basis of their PCR product expediency, as determined in previously performed conventional PCR assays. Two human DNA samples were used as templates for PCRs in which the CA or GATA repeats were amplified in separate tubes in the presence of the specific molecular beacons.
A spectrofluorometric thermal cycler possessing a 488-nm laser light source was used to determine the elicited fluorescence in real-time. In all the tested samples, fluorescent signals were generated with molecular beacons complementary to the products containing a specific STR, whereas a noncomplementary beacon does not generate a signal. In control reactions in which a DNA template is absent, no increase in fluorescence was observed (data not shown). Furthermore, agarose gel electrophoresis disclosed the presence of PCR products in all tested samples, except for the samples without any DNA template (control). Our results demonstrate that these molecular beacons bind specifically to their intended target product of either CA or GATA repeats.

\section{Real-Time Multiplex Detection of CA and GATA Repeat Products}

The final stage of our study was to establish a high-throughput application in which BAC clones containing CA and/or GATA repeats are distinguished in the same sealed reaction tube. This can be achieved by applying CA-TMR and GATA-TET molecular beacons simultaneously in a single PCR assay. The emission spectra of TET (emission maximum $535 \mathrm{~nm}$ ) and TMR (emission maximum $575 \mathrm{~nm}$ ) are reported during the annealing stage of all 35 thermal cycles. In the spectrofluorometer thermal cycler, the stored reference emission spectra of TET and TMR were utilized to decompose the recorded spectra into individual spectral contributions of GATA-TET and CA-TMR molecular beacons. The manifestation of green-yellow and orange fluorescence identifies the GATA repeat and CA repeat, respectively.

To examine the sensitivity of our newly developed detection assay, the 260 hybridization positive BAC clones, previously characterized by conventional PCR, were put to the test in a comparative molecular beacon assay. To preserve objectivity, a blind study was performed. BAC clone DNA, control human DNA, and nontemplate samples were mixed with the corresponding established primer pairs in Roswell Park Cancer Institute. These samples were then coded and shipped overnight to the Public Health Research Institute, where the molecular beacon genotyping data were generated.

Figure 3 shows the specificity of the real-time multiplex detection of CA and GATA repeat products. Five assays were carried out in parallel, initiated with BAC clone DNA containing CA and/or GATA repeats, BAC clone DNA without any CA/GATA repeats, and a nontemplate control. The result shows that individual clones are easily distinguished from each other by the color of the fluorescent signal generated by molecular beacons complementary to a CA or GATA repeat. No fluorescent signals were generated in both the template with no repeats and nontemplate control assays (data not shown).

The results in Table 1 show that molecular beacons detected 30 out of 43 GATA repeat clones (conventional PCR, 29/43) and 99 out of 162 CA repeat clones (conventional PCR, 92/ 162). With the CA-TMR and GATATET molecular beacons, we additionally verified $10 \mathrm{BAC}$ clones containing $\mathrm{CA}$ repeats and two BAC clones containing GATA repeats. However, one GATA repeat and three CA repeat clones, which were initially positively confirmed by the conventional method, were tested as negative. Unexpectedly, all eight positive clones anchored to locus D7S513, which is reputed to be a CA repeat marker, generated fluorescence with both TET and TMR fluorophore labeled beacons. A sequence query for marker D7S513 at the Whitehead Institute (http://www.genome. wi.mit.edu) showed that there were also 11 repeated GATA sequence blocks present within the product area of this locus. PCR products of BAC clones and positive control DNA containing ESTs or GGAA repeats, as well as the nontemplate controls, remained negative for TMR and/or TET fluorescence. Amplified positive control DNA, which contained CA and/or GATA repeats, did generate the expected TMR and/or TET fluorescence.

\section{DISCUSSION}

With the ambitious goals to com plete high-quality full DNA reference sequences of human (2) and mouse, 


\section{Research Report}

and ultimately those of other mammalian species (17), plants, and insects, there is a need to develop new supporting technologies. Resources of mapped BAC clones are prerequisites to sequencing entire genomes. Here, we describe a novel BAC clone confirmation assay by real-time PCR utilizing molecular beacons. This distinctive molecular beacon application is a highthroughput, PCR-based STS detection assay that will support the construction of a genome-wide arrayed BAC clone framework. So far, fluorescent fingerprint analysis (4) and PCR analysis (11) have always verified BAC clone eligibility. Visualization of individual PCR products by way of agarose gel electrophoresis is time consuming. Diametrically opposed to this technique is our newly developed PCR/hybridization test. This assay utilizing molecular beacons is simple, and it completely eradicates the labor-intensive product confirmation of gel electrophoresis. Furthermore, the specificity of the molecular beacon allows low-stringency amplification parameters, resulting in robust amplification of almost any primer/template combination. One of the many advantages of working with molecular beacons is that cross-contamination does not occur because tubes remain sealed while the data is being generated (19). The profound specificity of molecular beacons enables a multiplex one-tube detection assay because mixtures of multicolored molecular beacons can help discriminate between different targets $(9,18)$.

When 260 positive clones from the RPCI-11 human male BAC library (identified on high-density filter sets) were put through the conventional PCR confirmation test, 145 clones were verified (Table 1). On first sight, these results might seem to be rather poor. However, high-density filter sets used for hybridization screening purposes are reused to reduce the cost of analysis. Under initial conditions, some positive signals appeared to be previous hybridization signals (unpublished results). In addition, we also found that for $10 \%$ of STS/EST markers, the primer pairs were not successful in generating a PCR product. Since PCR was performed using established primer pairs for microsatellite markers, there is always a possibility that a marker sequence (derived from the Whitehead Center for Genome Research) either contains errors or polymorphic sites.

Due to the tremendous throughput of BAC clones that need confirmation, it is a necessity that PCR analyses for all the STSs were carried out under identical conditions. It is likely that optimizing the individual PCR conditions for markers that did not amplify could increase the percentage of positive clones. A consequence of the standard PCR conditions is the sometimes-ambiguous product production. Clone confirmation through size assessment of the PCR product by agarose gel electrophoresis often show vague or multiple bands (Figure 2). This makes interpretation of the results difficult, and repetitive screening becomes a necessity. This is in sharp contrast to molecular beacon assays. Due to the specific interaction of a molecular beacon with its complementary target, aspecific bands (PCR products) will be com pletely ignored.

The application described here of the real-time multiplex detection of STRs containing products in the same closed reaction vessel has proved to be an extremely sensitive and specific test system. This was accomplished by using two different molecular beacons simultaneously, each specific for a different STS region and each emitting light of a different color (TET, emission maximum $535 \mathrm{~nm}$; TMR, emission maximum $575 \mathrm{~nm}$ ). The results of this completely blind study (Table 1) show that with molecular beacons, 129 out of 205 BAC clones with CA or GATA repeats were detected, whereas with the conventional method, only 121 BAC clones could be verified. Furthermore, none of the $35 \mathrm{EST}$ or 20 of the BAC clones containing GGAA repeats were scored as false positive. The unanticipated detection of a combined CA and GATA repeat in all eight BAC clones anchored to locus D7S513 demonstrates the highly specific interaction of molecular beacons with their complementary target.

Although we only screened the two most common repeats observed in the STSs utilized in this study (CA and GATA repeats), the construction of 
multicolor molecular beacons specific for each other minor repeat element observed in STSs (GGAA, ATA, GGAT, GCT, etc.) could allow for the rapid, multiplex detection of any STS, from any organism, in a single assay. Fortuitously, the use of (multiple) molecular beacons can be highly cost effective. In particular, when used in large-scale projects, there will only be a marginal increase in expenses made per test as compared to the conventional agarose gel electrophoresis technique used to visualize individual PCR products. Recently, molecular beacons have been successfully applied in different mutation detection studies $(3,7,14,20,21)$. In conclusion, this newly developed molecular beacon application is perfectly suited to expedite the BAC clone confirmation throughput.

\section{ACKNOWLEDGMENTS}

The authors thank Drs. Jacqueline A.M. Vet for critical review of the man- uscript, Sanjay Tyagi for sharing his invaluable insights in the molecular beacons technology, Fred R. Kramer for his support, and Ms. Juliet A.E. Carter for her help with the graphical work. This study was supported by the National Institutes of Health National Human Genome Research Institute grant no. 1R01 HG01165-05, NIH grant no. HL43521, and the U.S. Department of Energy grant no. DE-FG02-94ER61883.

\section{REFERENCES}

1.Bonnet, G., S. Tyagi, A. Libchaber and F.R. Kramer. 1999. Thermodynamic basis of the enhanced specificity of structured DNA probes. Proc. Natl. Acad. Sci. USA 96:61716176.

2.Collins, F.S., A. Patrinos, E. Jordan, A. Chakravarti, R. Gesteland and L. Walters. 1998. New goals for the U.S. Human genome project: 1998-2003. Science 282:682-689.

3.Giesendorf, B.A.J., J.A.M. Vet, S. Tyagi, E.J.M.G. Mensink, F.J.M. Trijbels and H.J. Blom. 1998. Molecular beacons: a new approach for semiautomated mutation analysis. Clin. Chem. 44:482-486.

4.Gregory, S.G., G.R. Howell and D.R. Bentley. 1997. Genome mapping by fluorescent fingerprinting. Genome Res. 7:1162-1168.

5.Hillier, L.D., G. Lennon, M. Becker, M.F. Bonaldo, B. Chiapelli, S. Chissoe, N. Dietrich, T. DuBuque et al. 1996. Generation and analysis of 280,000 human expressed sequence tags. Genome Res. 6:807-828.

6.Hudson, T.J., L.D. Stein, S.S. Gerety, J. Ma, A.B. Castle, J. Silva, D.K. Slonim, R. Baptista et al. 1995. An STS-based map of the human genome. Science 270:1945-1954.

7.Kostrikis, L.G., S. Tyagi, M.M. Mhlanga, D.D. Ho and F.R Kramer. 1998. Spectral genotyping of human alleles. Science 279:12281229.

8.Kwok, S. and R. Higuchi. 1989. Avoiding false positives with PCR. Nature 339:237-238.

9.Marras, S.A.E., F.R. Kramer and S. Tyagi. 1999. Multiplex detection of single-nucleotide variations using molecular beacons. Genet. Anal. 14:151-156.

10.McPherson, J.D. 1999. Use of overlapping oligonucleotides (overgos) to generate hybridization probes, p. 209-213. In B. Birren, E.D. Green, P. Hieter, S. Klapholz, R.M. Myers, H. Riethman and J. Roskams (Eds.), Genome Analysis: A Laboratory Manual Series, Vol. 4: Mapping Genomes. CSH Laboratory Press, Cold Spring Harbor, NY.

11.Mullis, K., F. Faloona, S. Scharf, R. Saiki, G. Horn and H. Erlich. 1992. Specific enzymatic amplification of DNA in vitro: the polymerase chain reaction. 1986. BioTechnology 24:17-27.

12.Nowak, N.J., J. Conroy, G.P. Caldwell, J. Catanese, B. Trask, J.D. McPherson, D.R. Bentley, G. Shen and P.J. de Jong. 1999. A Resource of Mapped BAC Clones for Identifying Cancer Chromosome Aberrations. DOE Human Genome Program Contractor-Grantee
Workshop VII. January 12-16, Oakland, CA (Abstract). (http://genome.rtc.riken.go.jp/ hgmis/publicat/99santa/62.html)

13.Osoegawa, K., P.Y. Woon, B. Zhao, E. Frengen, M. Tateno, J.J. Catanese and P.J. de Jong. 1998. An improved approach for construction of bacterial artificial chromosome libraries. Genomics 52:1-8.

14.Piatek, A.S., S. Tyagi, A.C. Pol, A. Telenti, L.P. Miller, F.R. Kramer and D. Alland. 1998. Molecular beacon sequence analysis for detecting drug resistance in Mycobacterium tuberculosis. Nat. Biotechnol. 16:359-363.

15.Schuler, G.D., M.S. Boguski, E.A. Stewart, L.D. Stein, G. Gyapay, K. Rice, R.E. White, P. Rodriguez-Tome et al. 1996. A gene map of the human genome. Science 274:540-546.

16.Shizuya, H., B. Birren, U.J. Kim, V. Mancino, T. Slepak, Y. Tachiiri and M. Simon. 1992. Cloning and stable maintenance of 300kilobase-pair fragments of human DNA in Escherichia coli using an F-factor-based vector. Proc. Natl. Acad. Sci. USA 89:8794-8797.

17.Strausberg, R.L., E.A. Feingold, R.D. Klausner and F.S. Collins. 1999. The mammalian gene collection. Science 285:455-457.

18. Tyagi, S., D.P. Bratu and F.R. Kramer. 1998. Multicolor molecular beacons for allele discrimination. Nat. Biotechnol. 16:49-53.

19.Tyagi, S. and F.R. Kramer. 1996. Molecular beacons: probes that fluoresce upon hybridization. Nat. Biotechnol. 14:303-308.

20.Vet, J.A.M., A.R Majithia, S.A.E. Marras, S. Tyagi, S. Dube, B.J. Poiesz and F.R. Kramer. 1999. Multiplex detection of four pathogenic retroviruses using molecular beacons. Proc. Natl. Acad. Sci. USA 96:6394-6399.

21.Vogelstein, B. and K.W. Kinzler. 1999. Digital PCR. Proc. Natl. Acad. Sci. USA 96:92369241.

22.Zuker, M. 1989. On finding all suboptimal foldings of an RNA molecule. Science 244:4852.

Received 22 November 1999; accepted 12 July 2000.

Address correspondence to:

Dr. Rob C.A.A. van Schie

Department of Molecular and Cellular

Biophysics

Roswell Park Cancer Institute

Elm and Carlton Streets

Buffalo, NY 14263-0001, USA

e-mail:vanschie@roswellpark.org 